\title{
VARIABILIDADE NO ENVELHECIMENTO ATIVO SEGUNDO GÊNERO, IDADE E SAÚDE
}

\author{
Pricila Cristina Correa Ribeiro* \\ Anita Liberalesso Neri \\ Ana Paula Fabrino Bretas Cupertino" \\ Mônica Sanches Yassuda
}

\begin{abstract}
RESUMO. O presente estudo analisou a relação entre variáveis sociodemográficas, de saúde e participação em atividades físicas e ocupacionais. A amostra foi constituída de 155 idosos (60 anos ou mais) entrevistados pelo Estudo PENSA (Estudo dos Processos de Envelhecimento Saudável) na comunidade de Juiz de Fora/MG, sendo $28 \%$ homens e $72 \%$ mulheres, com média de 70,25 anos de idade ( $\mathrm{DP}=8,21)$. Para levantamento das variáveis analisadas foi utilizado o autorrelato. Observou-se que os homens eram mais participativos em atividades físicas e as mulheres em atividades ocupacionais, como tarefas de casa e voluntariado. Maior realização das atividades ocupacionais esteve associada à maior escolaridade, renda familiar e capacidade funcional. Diferentemente, a idade não se destacou como determinante de um estilo de vida ativo. Esse estudo demonstrou uma variabilidade de envelhecimento ativo na população estudada, segundo o gênero, e as variáveis sociodemográficas e de saúde, destacando as implicações do estilo de vida de idosos brasileiros no processo de envelhecimento saudável.
\end{abstract}

Palavras-chave: Idoso, envelhecimento, atividades.

\section{VARIABILITY IN ACTIVE AGING AS A FUNCTION OF GENDER, AGE AND HEALTH}

\begin{abstract}
The present study investigated the relation among socio-demographic and health variables and participation in physical and occupational activities. The sample was formed by 155 aged individuals (60 and older) interviewed by the PENSA study in Juiz de Fora, MG. $72 \%$ of the sample was female with average age of 70.3 years $(\mathrm{SD}=8.2)$. Questionnaires based on self-report were used for data collection. Results indicated that men participated more frequently in physical activities than women, and women were more active in occupational activities, such as housework and work as volunteers. Higher frequency of participation in occupational activities was associated with higher education, higher income, and higher functional status. However, age was not a significant determinant of active lifestyle. This present study demonstrated a variability of active aging due to gender, socio-demographic and health variables, highlighting the implications of the lifestyle of elderly Brazilians in the process of healthy aging.
\end{abstract}

Key words: Aged, aging, activities.

\section{VARIABILIDAD DEL ENVEJECIMIENTO ACTIVO SEGÚN GÉNERO, EDAD Y SALUD}

RESUMEN. El presente estudio ha analizado la relación entre las variables sociodemográficas de salud y la participación en actividades físicas y ocupacionales. La muestra fue de 155 ancianos (60 años o más) que fueron encuestados por el Estudio PENSA (Procesos de Envejecimiento Saludable) en la comunidad de Juiz de Fora/Minas Gerais, siendo $28 \%$ de hombres y $75 \%$ mujeres, con media de 70,25 años de edad ( $\mathrm{DP}=8,21)$. Se ha observado que los hombres eran más participativos en actividades físicas y las mujeres en actividades ocupacionales, como faenas de la casa y voluntariado. Gran parte de la realización de las actividades ocupacionales estuvo asociada a la mayor escolaridad, renta familiar y la capacidad funcional. Distintamente a eso, la edad no se ha destacado como determinante de un estilo de vida activo. Ese estudio demostró una variabilidad de envejecimiento activo en la población estudiada, según el género, la edad y la salud, destacando las implicaciones del estilo de vida de los ancianos brasileños para el proceso de envejecimiento saludable.

Palabras-clave: Anciano, envejecimiento,actividades.

\footnotetext{
* Mestre em Gerontologia pela UNICAMP, atualmente é doutoranda na Universidade Estadual de Rior de Janeiro-UERJ

\# Professora Titular da Universidade Estadual de Campinas-UNICAMP

II Professora assistente e pesquisadora da Kansas University Medical Center.

x Professora doutora da Escola de Artes, Ciências e Humanidade da Universidade de São Paulo-USP
} 
O envelhecimento ativo foi definido pela Organização Mundial de Saúde (OMS) (2002, p.13) como "o processo de otimização das oportunidades de saúde, participação e segurança, com o objetivo de melhorar a qualidade de vida à medida que as pessoas ficam mais velhas". O termo ativo refere-se, além da capacidade física e da participação na força de trabalho, ao envolvimento do indivíduo idoso nas questões sociais, econômicas, culturais, espirituais e civis. Diversos fatores determinantes da saúde no processo de envelhecimento foram relacionados ao estilo de vida ativo no envelhecimento - como cultura, gênero, promoção da saúde e prevenção de doenças, saúde mental, fatores psicológicos, hábitos de vida saudáveis, genética, fatores ambientais, apoio social, educação, fatores econômicos e trabalho. Os benefícios de um estilo de vida ativo e socialmente engajado são enfatizados pelas perspectivas teóricas da atividade (Birren \& Bengston, 1988) e do envelhecimento bem-sucedido.

O conceito de velhice bem-sucedida foi introduzido na Gerontologia por Havighurst em 1961 (Motta et al, 2005), no contexto do Kansas City Study of Adulthood and Aging e associava esse produto com a atividade, que gera satisfação e, consequentemente, preservação da saúde. Posteriormente, o conceito foi refinado por meio de dois modelos que hoje são dominantes na literatura gerontológica: o de Rowe e Kahn (1987) e o de Baltes e Baltes (1990). O conceito de velhice bem-sucedida tem forte componente cultural e é referido alternativamente como velhice saudável, ativa e produtiva, e também como boa qualidade de vida na velhice (Bass \& Caro, 2001). Embora os termos sejam igualmente sujeitos a críticas que apontam seu teor preconceituoso, dados internacionais e brasileiros convergem quanto aos seguintes aspectos, na definição do construto: a) físico: saúde física, capacidade funcional, capacidades de autocuidado, evitação de fatores de risco como álcool e tabaco e envolvimento com atividades físicas; b) social: engajamento em atividades de lazer, trabalho, satisfação com a carreira, suporte social, amigos e família e ter uma pensão adequada; c) emocional: satisfação com a vida geral e com a saúde em particular; d) pessoal: ter atitudes favoráveis ao envelhecimento e senso de controle e motivação para transmitir conhecimentos a pessoas mais jovens (Strawbridge, Wallhagen, \& Cohen, 2002, Fleck, Chachamovich, \& Trentini, 2003).

$\mathrm{Na}$ perspectiva de Rowe e Kahn (1987), o envelhecimento bem-sucedido é caracterizado pelo engajamento ativo com a vida associado à funcionalidade física e mental e à ausência de patologias. Esse engajamento ativo corresponde à manutenção das relações sociais e das atividades produtivas. Estas últimas compreendem diversas ocupações comuns entre idosos, como: atividades de auxiliar parentes; relacionar-se com vizinhos; realizar trabalhos voluntários; e atividades religiosas. Estes autores identificaram três fatores associados com a maior produtividade no envelhecimento: saúde física e capacidade funcional; características pessoais, como nível educacional e autoeficácia; e a participação nas relações sociais (Rowe \& Kahn, 1998).

Para explicar o ajustamento social do indivíduo diante das perdas na velhice, a teoria do desengajamento defendeu o afastamento social como um processo funcional para o idoso e para a sociedade. O desengajamento foi proposto como natural e espontâneo e a diminuição das interações e atividades sociais como um processo inevitável e inerente ao envelhecimento (Siqueira, 2002). A teoria da atividade surgiu como reação à teoria do desengajamento social, argumentando que, apesar das perdas físicas, psicológicas e sociais - comuns na velhice - o indivíduo idoso mantém suas necessidades psicossociais. Segundo esta perspectiva, a manutenção de atividades relaciona-se à satisfação e ao autoconceito positivo na velhice (Birren \& Bengston, 1988).

Controvérsias sobre a influência do aumento da idade no padrão de atividades são evidenciadas nos estudos empíricos. Aartsen et al. (2002), por exemplo, acompanharam por 6 anos 2.076 indivíduos residentes em Amsterdam e com idade entre 55-85 anos. Avaliaram a participação desta amostra em atividades como: serviços religiosos, associações de bairros, organizações de ajuda aos outros, cursos educacionais, esportes e atividades de lazer. Esse estudo apontou significativa diminuição da participação dos idosos nas atividades com o passar dos anos. Diferentemente, Newson e Kemps (2005) realizaram estudo longitudinal com 755 idosos do Australian Longitudinal Study on Ageing e demonstraram que a participação em atividades físicas e mentais diminuiu, enquanto a frequência em atividades sociais aumentou na amostra estudada. Destarte, mais investigações são necessárias para verificar quais atividades se intensificam e quais diminuem com o aumento da idade, bem como quais são os fatores capazes de mediar esta relação e explicar as modulações no envelhecimento ativo.

Assim, o estilo de vida ativo pode estar relacionado a diversos fatores, como saúde, ambiente e as características sociodemográficas. Dentre estas últimas, as variáveis gênero, idade e aspectos socioeconômicos mostraram-se 
influentes na escolha das atividades de estilo de vida entre idosos. Em estudo realizado por Aartsen et al. (2002) demonstrou-se que as mulheres realizavam com maior frequência as atividades cotidianas. Similarmente, na amostra estudada por Seeman et al. (2001) os homens engajavam-se mais em atividades físicas do que mulheres, as quais relataram mais envolvimento com as redes de contatos sociais. No engajamento em atividades físicas também se observam os efeitos do gênero e da idade. SalesCosta et al. (2003) sugerem que a prática de atividades está organizada segundo determinadas convenções, entre elas as concepções do ideal de corpo segundo o gênero. Em seu estudo com uma amostra de 4.614 adultos entre 20 e 60 anos, funcionários de uma universidade localizada no Estado do Rio de Janeiro, esses autores demonstraram que a inatividade física no tempo destinado ao lazer foi maior entre as mulheres, as quais se engajam mais em atividades individuais, que requerem menos força física, enquanto os homens preferem as atividades físicas coletivas e de caráter competitivo. Além disso, observou-se tendência de maior prática de atividades físicas entre as mulheres mais velhas, com maior escolaridade e com maior renda familiar.

Não obstante a relevância do estilo de vida ativo para o envelhecimento em suas dimensões biológicas e psicossociais, poucos estudos vêm buscando descrever o engajamento da população idosa em atividades gerais (de estilo de vida) e analisar a relação desse engajamento com aspectos sociodemográficos e/ou de saúde. No contexto brasileiro, ainda há compreensão limitada sobre a importância social e preventiva que o estilo de vida ativo pode ter para o envelhecimento. $\mathrm{O}$ padrão de engajamento em atividades entre os idosos precisa ser explorado para que ações interventivas possam ser planejadas com maior cautela.

Para suprir esta lacuna, o presente estudo investigou o engajamento em atividades em uma amostra de idosos residentes na comunidade, quantificando: a) prática de atividades físicas; b) realização de atividades instrumentais (trabalhos domésticos, jardinagem, compras e pagamentos); c) participação em atividades sociais (trabalho voluntário, cuidar dos netos, visitar parentes e amigos) e d) participação em atividades intelectuais (cursos, trabalhos manuais e entretenimentos culturais), relacionando estas atividades com gênero, idade, escolaridade e status socioeconômico e de saúde.

\section{MÉTODOS}

\section{Participantes}

O presente estudo foi desenvolvido a partir de dados coletados pelo Estudo PENSA (Estudo dos
Processos do Envelhecimento Saudável), um estudo de corte transversal realizado na cidade de Juiz de Fora/MG com o objetivo de descrever as características sociodemográficas, físicas, cognitivas e emocionais relacionadas ao envelhecimento saudável em uma amostra de idosos sistematicamente selecionada na comunidade. O estudo PENSA foi avaliado e aprovado pelo Comitê de Ética do Hospital Universitário de Juiz de Fora.

Para seleção da amostra a ser estudada pelo PENSA foram identificados os bairros de Juiz de Fora com percentual de idosos superior a $15 \%$, de acordo com os dados do CENSO 2000 realizado pelo Instituto Brasileiro de Geografia e Estatística (IBGE). Esses bairros foram visitados por recrutadores, que identificaram as residências com indivíduos acima de 60 anos. Aos idosos foi apresentado o estudo e feito o convite para participação na pesquisa. Aqueles que aceitaram participar assinaram um termo de livre consentimento e agendaram data posterior para entrevista, que foi realizada na própria residência do participante. Foram excluídos os sujeitos com déficits cognitivos, sensoriais e/ou físicos que os incapacitassem de compreender e responder ao protocolo do estudo.

As entrevistas foram realizadas pelos pesquisadores do estudo PENSA e por graduandos em psicologia. Para sistematizar a coleta dos dados e aumentar a objetividade da aplicação dos instrumentos, os entrevistadores foram treinados por meio de simulações de entrevistas e de testes-piloto realizados com idosos. O presente estudo utilizou dados coletados pelo estudo PENSA no período de março de 2002 a dezembro de 2003.

O estudo PENSA visitou 7.089 domicílios, nos quais 1.686 idosos foram identificados. Deste total, $6,9 \%$ estavam incapacitados, $24 \%$ recusaram-se a participar do estudo e $12 \%$ foram perdidos, isto é, assinaram o consentimento, mas não realizaram a entrevista. A amostra final do estudo foi de 956 idosos entrevistados na primeira fase do estudo, dos quais $28,2 \%$ eram homens e $71,8 \%$ eram mulheres, com idade entre 60 e 103 anos $(\mathrm{M}=72,4 \mathrm{DP}=8,3)$.

Em estudo anterior (Ribeiro, 2006), investigou-se a relação entre estilo de vida ativo e cognição, utilizando-se uma subamostra de 155 idosos do estudo PENSA que responderam à bateria cognitiva do CERAD e às questões referentes ao estilo de vida ativo. Esses participantes, selecionados ao acaso dentro da amostra original, foram convidados a responder à bateria do $\mathrm{CERAD}$, com o objetivo de verificar a aplicabilidade deste instrumento nessa população. O presente estudo valeu-se desta amostra. 


\section{Instrumentos}

Para alcançar os objetivos propostos foram utilizadas, do banco de dados do estudo PENSA, as variáveis que mensuraram dados sociodemográficos, estado de saúde e participação nas atividades estudadas. Os instrumentos utilizados encontram-se descritos a seguir:

- Questionário sociodemográfico: utilizado para descrever gênero, idade, estado civil, grau de escolaridade e renda familiar dos entrevistados.

- Saúde percebida: auto-avaliação do entrevistado sobre sua saúde de modo geral, classificando-a como: excelente, muito boa, boa, razoável ou péssima.

- Índice de Katz para Atividades básicas da vida diária (Gallo et al., 1995): escala composta de 6 itens para avaliação das atividades básicas de vida diária (ABVDs). Os escores variam entre 6 (independência total) e 18 (dependência total) para as ABVDs.

- Escala de Lawton e Brody (1969) para atividades instrumentais de vida diária: composta de 7 itens para avaliação das atividades instrumentais de vida diária (AIVDs). Cada atividade tem pontuação de 1 a 3, onde 1 indica independência total para realização da atividade, 2 indica dependência parcial e 3 dependência total. Assim, a pontuação total variou de 7 pontos (independência total) a 28 (dependência total) para as AIVDs.

- Atividades físicas: autorrelato sobre a frequência de prática de atividade física, segundo uma escala que variou entre: nunca, menos de duas vezes por semana, de duas a 4 vezes por semana, mais de 4 vezes por semana ou todos os dias. Verificou-se também quais os tipos de atividades físicas regularmente praticados.

- Atividades ocupacionais: foi utilizada uma tabela estruturada com as seguintes atividades: 1) sair para fazer compras e pagamentos; 2) realizar serviços domésticos; 3) sair para fazer visitas; 4) jardinagem; 5) cuidar de netos; auxiliar parentes; 6) participar de cursos como de línguas ou de artesanatos; 7) trabalhos manuais; 8) trabalho voluntário; 9) ir ao cinema, teatro; e 10) leituras. As atividades foram apresentadas ao entrevistado, que indicou se realizava tais atividades e com que frequência o fazia. A pontuação de cada atividade foi de 3 pontos quando a frequência de realização era diária; de 2 pontos para algumas vezes por semana, de 1 ponto para realização mensal e 0 quando a atividade não era praticada. $\mathrm{O}$ escore total das atividades ocupacionais variou de 0 a 30 pontos.

As atividades ocupacionais foram agrupadas por afinidade em subcategorias para facilitar a realização de algumas análises: a) instrumental (atividades 1; 2 e 4); b) social (atividades 3; 5 e 8); c) intelectual (atividades 6; 7; 9 e 10).
Para análise estatística foi utilizado o programa $S A S$ System for Windows (Statistical Analysis System). Para comparação das variáveis categóricas entre grupos foi utilizado o teste Qui-Quadrado ou, quando necessário, o teste exato de Fisher (presença de valores esperados menores que 5). Foram utilizados os testes de MannWhitney (2 grupos) e de Kruskal-Wallis (3 grupos) para comparar as variáveis numéricas entre grupos. Estes testes foram utilizados devido à ausência de distribuição normal das variáveis. O coeficiente de correlação de Spearman foi utilizado para verificar associação entre fatores socioeconômicos e atividades ocupacionais.

\section{RESULTADOS}

A amostra foi composta, em sua maior parte, de mulheres $(71,61 \%)$; e por grupos etários distribuídos em $53,6 \%$ de 60 a 69 anos, 33,5\% de 70 a 79 anos e $12,9 \%$ com 80 anos ou mais, sendo a média de idade de 70,25 anos $(\mathrm{DP}=8,21)$. Com relação ao estado civil, como esperado para esta coorte, houve predomínio de casados $(50,33 \%)$ e viúvos $(41,29 \%)$. Os solteiros representaram $3,87 \%$ e os divorciados ou separados, $4,52 \%$. Quanto ao status socioeconômico, observou-se que $17,53 \%$ relataram renda familiar entre um e dois salários mínimos; 35,06\% entre três e quatro salários; pouco mais de $24 \%$, renda entre 5 e 8 salários mínimos $(24,03 \%)$ e 23,38\% renda superior a oito salários mínimos. Quanto ao nível educacional, observou-se uma média de 6,73 anos de estudo ( $\mathrm{DP}=4,38$ ), sendo: 3,87\% dos idosos analfabetos; 5,81\% alfabetizados, mas sem nível primário completo; $43,87 \%$ com nível primário; 20,65\% com ginasial completo; $24,52 \%$ com ensino médio completo, e 1,29\% com formação superior.

Os indicadores socioeconômicos desta amostra revelaram-se superiores aos indicadores da população idosa brasileira. Segundo dados do IBGE (2005), 36,9\% da população brasileira com mais de 60 anos se constituíam de analfabeto ou de pessoas com menos de um ano de escolaridade em 2003; 25,2\% tinham entre 4 e 7 anos de estudo; 6,5\% tinham 11 anos de estudo; e 5,4\% estudaram por 12 anos ou mais. Quanto à renda familiar, observou-se que $26,6 \%$ das famílias brasileiras com idosos recebiam entre 1 e 2 salários mínimos e que apenas 8,4\% recebiam mais de 5 salários mínimos. Assim, os resultados apresentados devem ser interpretados com cautela, pois podem não ser representativos de outras regiões do país, com outras características sociodemográficas.

Com relação à avaliação subjetiva da saúde, observou-se que $8,39 \%$ classificaram sua saúde como excelente; $16,13 \%$ como muito boa; $44,52 \%$ como boa; $27,1 \%$ como razoável; e 3,87\% como péssima. Nos resultados da avaliação objetiva da saúde, a média da capacidade funcional foi de $6,32(\mathrm{DP}=1,64)$ para 
realização de ABVDs e 8,17 (DP = 2,58) para as AIVDs. Esse resultado revela que grande parte da amostra mostrou-se independente na maioria das AVDs.

No que se refere à participação dos idosos nas atividades ocupacionais, as análises descritivas mostraram que somente as seguintes atividades eram realizadas pela maioria da amostra: fazer visitas; compras e pagamentos; serviços domésticos; e leitura. Como pode ser visto na Tabela 1, a atividade de visitas foi a mais praticada diariamente. Compras e pagamentos eram mais realizados com frequência semanal, e os serviços de casa e atividade de leitura eram mais realizados mensalmente.

Tabela 1. Principais Atividades Ocupacionais Realizadas pela Amostra

\begin{tabular}{llc}
\hline \multicolumn{1}{c}{ Atividade } & Frequência de realização & \% \\
\hline \multirow{4}{*}{ Visitas } & Diária & 30.32 \\
& Semanal & 23.23 \\
& Mensal & 3.87 \\
& Não realizavam & 42.58 \\
\hline \multirow{4}{*}{ Compras e pagamentos } & Diária & 23.23 \\
& Semanal & 38.06 \\
& Mensal & 25.81 \\
& Não realizavam & 12.90 \\
\hline \multirow{4}{*}{ Serviços domésticos } & Diária & 1.29 \\
& Semanal & 19.90 \\
& Mensal & 63.87 \\
& Não realizavam & 21.94 \\
\hline \multirow{3}{*}{ Leitura } & Diária & 7.14 \\
& Semanal & 11.69 \\
& Mensal & 41.56 \\
& Não realizavam & 39.61 \\
\hline
\end{tabular}

As atividades menos realizadas foram: a) os cursos de línguas e artesanato (92,9\% dos idosos relataram não realizá-los); b) os trabalhos voluntários (85,81\% não os realizavam); c) as atividades culturais
(81,29\% não as realizavam); d) os trabalhos manuais (73,55\% não os realizavam); e) a jardinagem (não praticada por $70,32 \%$ dos idosos).

Quando as atividades ocupacionais foram agrupadas em instrumentais, sociais e intelectuais, foram obtidos os seguintes escores de participação: média de 3,11 pontos $(\mathrm{DP}=1,51)$ para as atividades instrumentais; média de 2,3 para as atividades sociais $(\mathrm{DP}=1,88)$; e média de 2,02 $(\mathrm{DP}=1,98)$ para as atividades intelectuais, lembrando que os escores para cada atividade variaram entre 0 (nunca realiza) e 3 (realiza diariamente). No escore total de participação nas atividades ocupacionais a média obtida pela amostra foi de 7,43 (DP=3,82) pontos, sendo que para cada participante essa média poderia variar entre 0 (não realiza atividade alguma) e 30 (realiza as 10 atividades diariamente). A amostra estudada mostrou, portanto, maior engajamento em atividades instrumentais de vida diária e menor engajamento em atividades intelectuais e sociais, como cursos, trabalhos voluntários e entretenimentos culturais.

Quanto à prática das atividades físicas, observouse que $48,39 \%$ da amostra relataram nunca fazer exercício físico; $6,45 \%$ praticavam exercícios pelo menos duas vezes por semana; $16,77 \%$ o faziam de duas a quatro vezes por semana; 5,16\% faziam exercícios mais de quatro vezes por semana e 23,23\% os faziam diariamente. A caminhada foi o tipo de exercício físico mais praticado $(36,13 \%)$, e entre os outros tipos de exercício realizados pela amostra foram registradas a ginástica e a hidroginástica, praticadas por pouco mais de $5 \%$ dos idosos: $5,81 \%$ e $5,16 \%$, respectivamente.

Nas comparações do estilo de vida ativo entre os gêneros foram encontradas diferenças significativas na frequência de realização de atividades físicas e de todas as atividades ocupacionais investigadas, como mostra a Tabela 2.

Tabela 2. Comparação dos Escores de Participação nas Atividades entre Gêneros

\begin{tabular}{lcccccccc}
\hline \multirow{2}{*}{ Atividades } & \multicolumn{3}{c}{ Masculino } & & & Feminino & \multicolumn{2}{c}{$\begin{array}{c}\text { Estatística } \\
\text { Mann-Whitor* }\end{array}$} \\
\cline { 2 - 6 } & Min. & Mediana & Máx. & Min. & Mediana & Máx. & 0.003 \\
Físicas & 1.00 & 3.00 & 5.00 & 1.00 & 1.00 & 5.00 & $\mathrm{U}=1743.5 ; \mathrm{Z}=2.97$ \\
Instrumentais & 0.00 & 3.00 & 5.00 & 0.00 & 3.00 & 7.00 & $\mathrm{U}=1932.0 ; \mathrm{Z}=2.07$ & 0.038 \\
Sociais & 0.00 & 1.00 & 4.00 & 0.00 & 3.00 & 9.00 & $\mathrm{U}=1614.0 ; \mathrm{Z}=3.35$ & $<0.001$ \\
Intelectuais & 0.00 & 1.00 & 4.00 & 0.00 & 2.00 & 9.00 & $\mathrm{U}=1711.5 ; \mathrm{Z}=2.96$ \\
Ocupacionais & 0.00 & 5.00 & 11.00 & 1.00 & 8.00 & 22.00 & $\mathrm{U}=1403.0 ; \mathrm{Z}=4.14$ \\
\hline
\end{tabular}

* significativo para $\mathrm{p}<0,05$

Observou-se que a atividade física é mais frequentemente realizada pelos homens. Nas análises comparativas da atividade física como variável categórica, verificou-se que $43,18 \%$ dos homens relataram praticar exercícios físicos diariamente e que entre as mulheres somente $15,32 \%$ têm essa atividade 
como prática diária e $54 \%$ relataram não realizar atividades físicas. Não obstante, a prática das atividades ocupacionais (instrumentais, sociais e intelectuais) é mais comum entre as mulheres, que alcançaram médias maiores de frequência de realização dessas atividades (8,23 pontos) em comparação à média da amostra masculina $(5,43$ pontos).

Em relação à faixa etária e à realização de atividades, não houve diferença significativa entre os grupos na prática das atividades físicas $(\mathrm{H}=1.36$; $\mathrm{p}=0.507)$, sociais $(\mathrm{H}=0.50 ; \mathrm{p}=0.778)$ e/ou intelectuais $(\mathrm{H}=0.40 ; \quad \mathrm{p}=0.818)$. Observou-se diferença significativa nas atividades instrumentais $(\mathrm{H}=6.82$; $\mathrm{p}=0.033$ ), que eram realizadas com menor frequência pelos idosos com 80 anos ou mais, quando comparados aos grupos de 60-69 anos e de 70-79 anos.

No que se refere aos fatores socioeconômicos (escolaridade e renda), observou-se correlação estatisticamente significativa, entretanto de magnitude modesta, entre a quantidade de anos de estudos e a frequência de realização das atividades intelectuais ( $\mathrm{r}$ $=0.308 ; \mathrm{p}<0.001)$ e das atividades ocupacionais $(\mathrm{r}=$ $0.164 ; \mathrm{p}=0.04)$. Similarmente, verificou-se correlação entre a renda familiar e as atividades intelectuais $(r=0.249 ; p=0.002)$ e ocupacionais $(r=0.196 ; p=0.02)$, indicando que os indivíduos mais escolarizados e com maior renda relataram maior participação nas atividades culturais e de leitura e nos cursos (línguas e artesanato), além de maior escore total na realização das atividades ocupacionais. Não foram encontradas relações estatisticamente significativas entre o status socioeconômico e as demais atividades investigadas.

Com relação aos aspectos de saúde, as análises de comparações mostraram diferenças estatisticamente significativas para a frequência de prática das atividades físicas de acordo com a saúde percebida $\left(\chi^{2}=16.63 ; \mathrm{p}=0.002\right)$. Os idosos que avaliaram sua saúde como excelente ou muito boa praticavam atividade física com maior frequência do que aqueles que avaliaram sua saúde como razoável ou péssima. Os resultados mostraram que $40 \%$ dos idosos que classificaram sua saúde como excelente ou muito boa relataram praticar exercícios físicos diariamente, $26 \%$ até quatro vezes por semana, e $34 \%$ referiram não praticar exercícios. Dos idosos que classificaram sua saúde como boa, 27\% mantinham prática diária de atividades físicas, $25 \%$ praticavam-nas até quatro vezes por semana e $48 \%$ nunca faziam exercícios; e dos idosos que consideraram a saúde como razoável ou péssima, apenas $4 \%$ praticavam exercícios físicos diariamente; $36 \%$ o faziam até quatro vezes por semana e $60 \%$ relataram não realizar atividades físicas. Para as atividades ocupacionais nenhuma diferença significativa em relação à saúde percebida foi observada.

A capacidade funcional e a realização de atividades físicas mostraram-se negativamente correlacionadas, ou seja, quanto maior o escore para ABVDs ( $\mathrm{r}=-0.159 ; \mathrm{p}=0.047)$ e AIVDs ( $\mathrm{r}=-0.211$; $\mathrm{p}=0.047$ ) (pontuação alta revela maior dependência) menor a frequência de realização de exercícios físicos.

$\mathrm{O}$ escore de funcionalidade mostrou-se, ainda, associado negativamente ao escore total de atividades ocupacionais $(\mathrm{r}=-0.214 ; \mathrm{p}=0.001)$; entretanto é necessário considerar que há sobreposição entre as questões de funcionalidade e de atividades ocupacionais, as quais, com exceção da atividade de jardinagem, investigam a realização de atividades similares (realizar serviços domésticos, compras e pagamentos), o que poderia explicar a associação encontrada. A correlação sugere que quanto maior a dependência funcional menor a frequiência de atividades ocupacionais. Verificou-se associação negativa entre as perdas em AIVDs e a frequência de atividades sociais $(r=-0.158 ; p=0.048)$.

\section{DISCUSSÃO}

Os resultados deste estudo mostraram um perfil diferente de estilo de vida ativo quando comparados os gêneros. Análises de correlação também indicaram uma associação significativa entre escolaridade, renda e estado de saúde autorrelatada e a frequência de realização de atividades. Os dados demonstram, portanto, que há variabilidade no padrão de envelhecimento ativo nesta amostra e que esta variabilidade associa-se a diversas características sociodemográficas.

No que se refere ao gênero, observou-se que as mulheres realizavam com mais frequência as atividades ocupacionais, provavelmente fruto de um efeito de coorte, no qual cabe às mulheres dedicaremse às atividades de âmbito social e instrumental como tarefas domésticas, trabalhos voluntários e cuidados e auxílios aos netos/parentes. Por outro lado, os homens praticavam com maior frequência as atividades físicas. Estes resultados estão em concordância com diversas pesquisas (Aartsen et al. 2002; Seeman et al., 2001; Zunzunegui et al. 2003), contudo divergem do estudo de Scarmeas et al. (2001), no qual as mulheres norteamericanas apresentaram menor escore de participação em atividades ocupacionais quando comparadas aos homens. 
Os resultados da presente investigação não permitem uma generalização quanto ao tipo de atividade praticada por gênero, mas apontam a necessidade de uma diferenciação entre os gêneros, uma vez que homens e mulheres podem apresentar padrões diferentes de envelhecimento ativo e de preferência por tipos de atividade, resultando em efeitos divergentes no processo de envelhecimento. Supõe-se, então, que o contexto histórico e cultural influencia o estilo de vida do indivíduo, determinando seu desenvolvimento da infância até a velhice. Assim, corrobora-se o pressuposto que o processo de envelhecimento está relacionado não só aos fatores biológicos e psicossociais, mas também ao contexto cultural e sócio-histórico no qual o indivíduo está inserido e ao qual tem que se adaptar (Baltes \& Singer, 2001). Estudos de corte sequenciais podem verificar as hipóteses levantadas sobre o efeito de coorte no estilo de vida ativo dos idosos.

$\mathrm{Na}$ amostra do presente estudo os indivíduos com maior escolaridade e maior renda mostraram-se mais engajados em atividades intelectuais, o que pode ser resultado do maior acesso aos entretenimentos culturais e aos cursos (línguas e outros) associado ao status socioeconômico. Este resultado está de acordo com os achados de outros estudos. Scarmeas et al. (2001), por exemplo, encontraram relação estatisticamente significativa entre escolaridade e participação de idosos em atividades de lazer. O estudo acompanhou por sete anos uma amostra de 1.772 norte-americanos com 65 anos ou mais, demonstrando que entre os idosos com nível de escolaridade maior a média de realização de atividades de lazer também foi maior, se comparados aos indivíduos com menor escolaridade. Dessa forma, os indicadores sociais (renda e escolaridade) são vistos como determinantes do estilo de vida, constituindo-se em variáveis significativas para a investigação da variabilidade do envelhecimento ativo.

No que se refere à idade, embora alguns estudos tenham demonstrado que há uma diminuição do nível de atividade relacionada ao aumento da idade (Aartsen et al., 2002), no presente estudo, na maioria das atividades o menor engajamento não esteve relacionado com maior idade, o que está em concordância com a teoria da atividade que argumenta sobre a necessidade do idoso de manter os níveis de atividade apesar das perdas associadas ao envelhecimento (Birren \& Bengston, 1988). Não obstante, com o aumento da idade houve diminuição significativa na frequência de realização das atividades instrumentais, como fazer compras e realizar serviços domésticos; ou seja, indivíduos mais velhos praticavam com menor frequência tais atividades, o que pode ser explicado pelo aumento do risco de fragilidade e perda da independência para realização destas tarefas. Dessa forma, supõe-se que não seja o aumento da idade isoladamente, mas, principalmente, a diminuição das capacidades físicas o que determina o menor engajamento do idoso em um estilo de vida ativo. Em concordância com esta argumentação, alguns estudos demonstraram que melhor estado de saúde associou-se ao maior engajamento em diversas atividades nos indivíduos idosos (Aartsen et al., 2002; Wang et al., 2002).

No presente estudo utilizou-se a metodologia de corte transversal e uma amostra pequena. Por isso estudos epidemiológicos precisam ser realizados para possibilitar a generalização dos dados observados e a ampliação do conhecimento sobre variáveis de atividade e seus fatores determinantes na população idosa brasileira.

Para mensuração do estilo de vida ativo foi utilizado, nesta pesquisa, o autorrelato, com o objetivo principal de descrever, de forma geral, como os idosos se ocupam em seu dia-a-dia. Sugere-se que futuras pesquisas mensurem o tempo que o indivíduo gasta com cada atividade, e não somente se a realiza todos os dias, semanal ou mensalmente. Embora seja frequente o uso do autorrelato para medir o que os indivíduos realizam em seu cotidiano, por ser este um método simples de mensuração ecológica do estilo de vida ativo, escalas de atividade também podem ser empregadas nas investigações de envelhecimento ativo (Clark \& Bond, 1995). Não obstante, no contexto brasileiro instrumentos desse tipo ainda precisam ser validados ou construídos para mensuração do estilo de vida da população idosa.

Além dos avanços empíricos, são necessárias ampliações teóricas sobre o estilo de vida e o envelhecimento ativo, pois a definição das atividades varia largamente não apenas na mensuração utilizada, mas também no nível conceitual. As pesquisas sobre envelhecimento ativo devem ser ampliadas de forma a explorar os diversos fatores incluídos neste conceito. No presente estudo, por exemplo, não foram analisadas as dimensões da participação dos idosos nas questões econômicas e espirituais e na força de trabalho, consideradas como importantes aspectos do envelhecimento ativo (OMS, 2002). O trabalho como fonte de demanda cognitiva e estimulação mental deve ser estudado, uma vez que se associa à complexidade ambiental, sendo necessário compreender que tipo de trabalho desempenhado pelo indivíduo está relacionado ao maior benefício para a funcionalidade cognitiva na velhice (Rowe \& Kahn, 1987). A 
participação em atividades religiosas também é de fundamental importância, pois estudiosos vêm demonstrando que as práticas religiosas e a espiritualidade podem influenciar no bem-estar na velhice (Sommerhalder \& Goldstein, 2006). Outra dimensão que deve ser estudada é a rede de suporte social e emocional do idoso, também associada à saúde física, emocional e cognitiva e ao estilo de vida ativo na velhice (Fratiglioni et al., 2000; Seeman et al., 2001).

$\mathrm{O}$ presente estudo apontou que fatores sociodemográficos estão associados a diferentes padrões de realização de atividades instrumentais, sociais e intelectuais. De modo geral, gênero feminino, melhor saúde autorrelatada, maior funcionalidade, renda e escolaridade estão associadas a maior frequência de atividades. A relação entre atividade e idade foi tênue, pois somente se detectou menor realização de atividades instrumentais em pessoas acima de 80 anos. Sugere-se que futuras investigações deem continuidade ao levantamento do estilo de vida dos idosos brasileiros residentes na comunidade e que avancem a investigação sobre o conceito de envelhecimento ativo e suas implicações no bem-estar físico e psicológico dessa população.

\section{REFERÊNCIAS}

Aartsen, M.J.; Smits, C.H.M.; Tiburg, T.; Knipscheer, K.C.P.M; \& Deeg, D. J.H. (2002). Activity in older adults: cause or consequence of cognitive functioning? A longitudinal study on everyday activities and cognitive performance in older adults. Journal of Gerontology: Psychological Sciences. 57B(2), 153162.

Baltes, P.B \& Baltes M.M. (1990). Psychological perspectives on successful aging: The model of selective optimization with compensation. In P. B. Baltes, \& M. M. Baltes (Eds), Successful Aging: Perspectives from the Behavioral Sciences (pp. 1-34). NY: Cambridge University Press.

Baltes, P.B. \& Singer, T. (2001). Plasticity and the ageing mind: an exemplar of the bio-cultural orchestration of brain and behaviour. European Review. 9(1), 59-76.

Bass, S.A. \& Caro, F.G. (2001). Productive aging: A conceptual framework. In N. Morrow, J. Hinterlong \& M. Sherraden (Eds.), Productive aging: concepts and challenges (pp. 37-70) Baltimore, Maryland: The John Hopkins University Press.

Birren, J.E. \& Bengston, V.L. (1988). Emergent theories of aging (pp. 333-355). New York: Springer.

Clark, M.S. \& Bond, M.J. (1995). The Adelaide Activities Profile: a measure of the life-style activities of elderly people. Aging and Clinical Experimental Reserch, 7, 174-184.

Fleck, M.A., Chachamovich, E., Trentini, C.M. (2003). Projeto WHOQOL-OLD: método e resultados de grupos focais no Brasil. Rev Saúde Pública, 37, 793-799.
Fratiglioni, L.; Wang, H.; Ericsson, K.; Maytan, M.; \& Winblad, B. (2000). Influence of social network on ocorrence of dementia: a community-based longitudinal study. Lancet, 355, 1315-19.

Gallo, J.J.; Reichel, W. \& Andersen, L.M. (1995). Handbook of Geriatric Assessment 2. ed. Aspen Publishers, Gaithersburg, Maryland.

Instituto Brasileiro de Geografia e estatística (2005). Síntese de Indicadores Sociais 2004. Rio de Janeiro: IBGE

Lawton M.P. \& Brody E.M. (1969) Assessment of older people: selfmaintaining and instrumental activities of daily living. Gerontologist. 9(3),179-86.

Motta, M., Bennati, E., Ferlito, L., Malaguarnera, M., Motta, L., \& Italian Multicenter Study on Centenarians (IMUSCE). (2005). Successful aging in centenarians: Myths and reality. Archives of Gerontology and Geriatrics, 40, 241-251.

Newson, R.S. \& Kemps, E.B. (2005). General lifestyle activities as a predictor of current cognition and cognitive change in older adults: a cross-sectional and Longitudinal examination. Journal of gerontology: psychological sciences. 60B(3), 113-120.

Ribeiro, P.C.C. (2006). Estilo de vida ativo no envelhecimento e sua relação com o desempenho cognitivo: um estudo com idosos residentes na comunidade. Dissertação de Mestrado Nãopublicada, Curso de Pós-graduação em Gerontologia, Universidade Estadual de Campinas. Campinas, SP. 115p.

Rowe, J.W. \& Kahn, R.L. (1987). Human aging: usual and successful. Science. 237, 143-149

Rowe, J. W. \& Kahn, R. L. (1998). Successful aging. New York: Pantenon Books.

Organização Mundial da Saúde (OMS) (2002). Envelhecimento ativo: uma política de saúde / World Health Organization. (S, Gontijo Trad.), Brasília: Organização Pan-Americana da Saúde. 60p. Disponível em: http://dtr2001.saude.gov.br/svs/pub/ pdfs/envelhecimento_ativo.pdf

Salles-Costa, R. H.M.L.; Werneck , G.L.; Faerstein E.; Lopes, C.S. (2003) Gênero e prática de atividade física de lazer. Caderno de Saúde Pública. 19 (suppl.2), S325-S333

Scarmeas, N.; Levy, G.; Tang, M.-X.; Manly, J.; \& Stern, Y. (2001). Influence of leisure activity on the incidence of Alzheimer's Disease. Neurology, 57(12).

Seeman, T.E.; Lusignolo, T.M.; Albert, M; Berkman, L. (2001). Social relationships, social support, and patterns of cognitive aging in healthy, high-functioning older adults: MacArthur studies of successful aging. Health psychology. 20(4), 243-255.

Siqueira, M.E.C. (2002). Teorias Sociológicas do Envelhecimento. Em: Freitas, E.V.; Py, L. et. al (Org) Tratado de Geriatria e Gerontologia (pp.47-58). Rio de Janeiro: Guanabara.

Sommerhalder, C. \& Goldstein, L. L. (2006). O papel da religiosidade e da espiritualidade na vida adulta e na velhice. Em: Freitas, E.V; PY, L.et al (Org.) Tratado de Geriatria e Gerontologia. Rio de Janeiro: SBGG e Guanabara Koogan, 2 Ed. Revisada e Ampliada.

Strawbridge, W.J; Wallhagen, M.I. \& Cohen, R.D (2002). Successful Aging and Well-Being: Self-Rated Compared With Rowe and Kahn. Gerontologist, 42, $727-733$.

Wang, H.; Karp, A.; Winblad, B.; \& Fratiglioni. L. (2002). Late-Life Engagement in Social and Leisure Activities Is Associated with a Decreased Risk of Dementia: A Longitudinal Study from the Kungsholmen Project. Am J Epidemiol. 155(12), 1081-1087. 
Zunzunegui, M. V.; Alvorado, B.E.; Ser, T.D.; \& Otero, A. (2003). Social networks, social integration, and social engagement determine cognitive decline in community-dwelling spanish older adults. Journal of gerontology. 58B(2), 93-100.

Endereço para correspondência : Mônica Sanches Yassuda, EACH USP, Gerontologia. Av. Arlindo Bettio, 1000, Ermelino Matarazzo, CEP 03828-000,São Paulo-SP, Brasil. E-mail: yassuda@usp.br 